\title{
APRENDIZAJE LIDERADO POR LOS ESTUDIANTES MEDIANTE ELABORACIÓN DE VÍDEOS COLABORATIVOS SOBRE HIDROLOGÍA Y EROSIÓN
}

\section{STUDENT-LED LEARNING THROUGH COLLABORATIVE VIDEOS ON HYDROLOGY AND SOIL EROSION}

\author{
Vanwalleghem, T.*, Martínez, G., \\ Román, A., García, V., \\ Laguna, A., Giráldez, J.V., Peña, A. \\ *ag2vavat@uco.es
}

\section{Escuela Técnica Superior de Ingeniería Agronómica y de Montes, Universidad de Córdoba}

Received: 30/06/2017_Accepted: 27/06/2018

\begin{abstract}
This study aims to give an impulse to the modernization of the learning process through a collaborative effort, led by students themselves. Through the combination of Information and Communication Technologies (ICT) and new social media used frequently by students (Youtube, Instagram, Pinterest,...) the learning process was enhanced while at the same time promoting learning through new technological approaches that helped to analyze and understand processes related to hydrology and soil erosion. Through the elaboration of their own videos own different topics chosen by student groups, they identify the learning objectives of the course subject according to its importance and assume responsibilities of their own learning process.

The chosen course themes were: (i) the movement of the wetting front in soils of different texture; (ii) water retention in green roofs; (iii) field water infiltration practical. The students, in groups of 2 to 6 were assigned to design the scenario for the videos, shooting and finally its diffusion through moodle and social media (in this case Twitter was selected). This collection of educative videos will constitute a new learning tool for following courses. It contributed to the group to deepen its knowledge about a particular course theme, and show their progress to their fellow students so that the learning process would be collective.
\end{abstract}

Keywords: ICT, videos, collaborative learning, competency, hydrology

\section{Resumen}

Este trabajo pretende dar un impulso a la modernización educativa mediante un esfuerzo colaborativo dirigido por los propios estudiantes. Mediante la combinación de las Tecnologías de la Información y la Comunicación (TIC) y las nuevas redes sociales usadas frecuentemente por los estudiantes (Twitter, Periscope, Youtube, Instagram, Pinterest por citar algunos) se intenta facilitar la enseñanza junto con el aprendizaje a través de nuevos enfoques tecnológicos que ayuden a analizar y comprender los procesos hidrológicos y erosivos en el suelo. Mediante la elaboración de sus propios vídeos sobre diferentes temas elegidos por los propios estudiantes, éstos identifican los objetivos del aprendizaje en la materia según su importancia y asumen cierta responsabilidad de su propio proceso de aprendizaje .

Los temas elegidos han sido: (i) el movimiento del frente de humedad en diferentes tipos de suelo; (ii) la retención de agua en las cubiertas vegetales sobre edificios, conocidas también como techos verdes; y (iii) la infiltración de agua en el suelo desde una perspectiva más general. Los estudiantes, en grupos de 2 a 6 personas, han diseñado un guión para los vídeos, han filmado los vídeos y, finalmente, los han alojado en el espacio de la plataforma moodle y en redes sociales (en particular en Twitter). Esta colección de vídeos constituye una nueva herramienta de enseñanzaaprendizaje, que permite primero a los distintos grupos de estudiantes profundizar en su aprendizaje sobre un tema en concreto, y después enseñar sus progresos al resto de estudiantes de los demás grupos para un aprendizaje del colectivo.

Palabras clave: TIC, videos, enseñanza-aprendizaje colaborativa, trabajo por competencias, hidrología

\section{INTRODUCCIÓN}

El estudio de la interfaz agua-suelo-planta es esencial en las titulaciones del Grado de Ingeniería Forestal y del Grado de Ingeniería Agroalimentaria y del Medio Rural, así como del Máster en Ingeniería Agronómica y del Máster en Ingeniería de Montes, por ser el suelo la base de la vida y un depósito esencial en el ciclo del agua en la Naturaleza. Por ello, las asignaturas relacionadas con Hidrología y Erosión tienen mucha importancia en el curriculum de estos estudiantes. En estas materias hay muchos procesos que, interactúan entre sí, como la infiltración de agua en el suelo, la generación de la escorrentía superficial, la recarga de acuíferos y la erosión del suelo, siendo arduo su aprendizaje.

La exposición de estos procesos en una clase magistral no está exenta de dificultades. Se trata de procesos complejos que requieren un esfuerzo apreciable de comprensión, en especial, en un aula cerrada, sin más ayuda que la explicación del docente y unos medios audiovisuales en un intervalo reducido de tiempo. Por ello, desde hace unos años, y en diferentes asignaturas, se implantaron prácticas y visitas de campo.

Este artículo pretende impulsar nuevos métodos docentes integrando clases prácticas y visitas de campo con las Tecnologías de la Información y la Comunicación (TIC), así como facilitar la enseñanza y el aprendizaje a través de un nuevo enfoque para desarrollarlos en un proceso compartido por docente y estudiante. Por ello, se propone que los estudiantes elaboren unos documentos visuales, vídeos, para explicar algunos procesos de Hidrología y Erosión a elegir entre diferentes propuestas ofrecidas por el profesorado. Algunos ejemplos son: (i) el movimiento del frente de humedad en suelos de distinta 
porosidad, con interfaces de poros gruesos, poros finos, incluyendo obstáculos como elementos gruesos, raíces, fisuras y grietas, e incluso desarrollo de hidrofobicidad en los agregados del suelo. Gardner y Hsieh desarrollaron un vídeo en los años sesenta del pasado siglo titulado 'Water movement in soils' que sigue atrayendo la atención de docentes y estudiantes de estas asignaturas.; (ii) succión del agua por raíces vegetales; (iii) erosión por impacto de gotas, o por esfuerzo de corte debido a la escorrentía en suelos con o sin cobertura vegetal, (iii) la retención de agua en cubiertas vegetales y en techos verdes entre otros.

Para este fin los estudiantes, en grupos de 2 a 6 personas, preparan el guión del video, su elaboración, y, finalmente, los han alojado en el espacio de la plataforma moodle y en redes sociales (en particular en Twitter). Se pretende que esta colección de vídeos sea una nueva herramienta que permita primero al propio grupo el aprendizaje al profundizar en un tema en concreto, y después la enseñanza a toda la clase del trabajo del grupo. Y así que el resultado sea por una parte la adquisición de la información, y por otra parte su difusión compartiéndola con el resto de estudiantes y otras personas interesadas a través de las redes sociales. En este método el proceso de aprendizaje es prioritario, y su contenido, siendo también importante es algo más secundario. De este modo, los estudiantes aprenden a plantearse un problema típico de la hidrología, a buscar una solución, a desarrollarla, y, finalmente, a divulgar los resultados. Los grupos serán siempre de carácter mixto, promocionando el carácter paritario de los mismos. Con los resultados del presente artículo, se pretende que se amplíen la aplicación práctica de los conocimientos teóricos adquiridos en clase, la participación activa y continua durante el curso, la autonomía e iniciativa personal, el trabajo en equipo y el desarrollo de capacidades que permitan al estudiante construir razonadamente el desarrollo de una materia concreta y a la vez solucionar problemas en un futuro inmediato tanto en el ámbito científico como en el profesional.

De esta forma, con el desarrollo de esta colección de casos, se pretende el perfeccionamiento en el estudio de la Hidrología y Erosión, potenciando las siguientes competencias en el alumnado:

- Puesta en práctica de los conocimientos teóricos adquiridos a través del análisis de casos reales.

- Desarrollo de la capacidad de síntesis de los conocimientos teóricos y gestión la información obtenida

- Participación de los estudiantes de forma activa y continua durante el curso académico en un trabajo en grupo, donde se compartan los conocimientos adquiridos y se fomente la autonomía e iniciativa personal, el intercambio de nuevas ideas, incluyendo el "aprender a aprender", que constituyen pilares fundamentales para su formación académica y su posterior aprovechamiento en ámbitos laborales, incluyendo la investigación.

- Desarrollo de capacidades que le permitan construir razonadamente y a la vez solucionar problemas en un futuro inmediato tanto en el ámbito científico como profesional.

\section{OBJETIVOS}

El objetivo general es el desarrollo de un sistema de aprendizaje y enseñanza basado en una colección de vídeos elaborados por los propios estudiantes y basados en ejercicios prácticos relacionados con la hidrología y la erosión.

\section{METODOLOGÍA}

\subsection{DESARROLLO DE LOS MODELOS A ESCALA}

La metodología a seguir se divide en 3 actividades detalladas a continuación, siguiendo el modelo de la metodología de aprendizaje colaborativa (Bruffee, 1998):

Actividad 1. Elección del tema a desarrollar por el alumnado y preparación de contenidos

Los grupos de estudiantes, de entre 2 y 4 personas cada uno, eligen un tema, buscan la información bibliográfica pertinente, incluyendo casos prácticos relacionados, y diseñan el guión del vídeo. Durante esta fase, el profesorado aconseja y orienta, sin restringir la libertad del estudiantado para la elección del tema y el diseño de sus propios objetivos de aprendizaje 
Actividad 2. Elaboración del vídeo.

La primera parte de esta actividad es la descripción del ejercicio práctico basado en un proceso hidrológico o erosivo. Los estudiantes pueden usar el material de laboratorio y de campo disponible del grupo de investigación AGR-127 (Hidrología e hidráulica agrícola), para elaborar el correspondiente vídeo. Aparte, en este artículo se propone la elaboración de 3 modelos a escala para su uso específico, y basado en los ejemplos de la Figura 1:

(1) modelo a escala en metacrilato para simular y medir la erosión de suelo en diferentes tipos de suelos y con diferentes coberturas

(2) modelo a escala para simular deslizamientos de suelo

(3) modelo a escala para simular el comportamiento de techos verdes

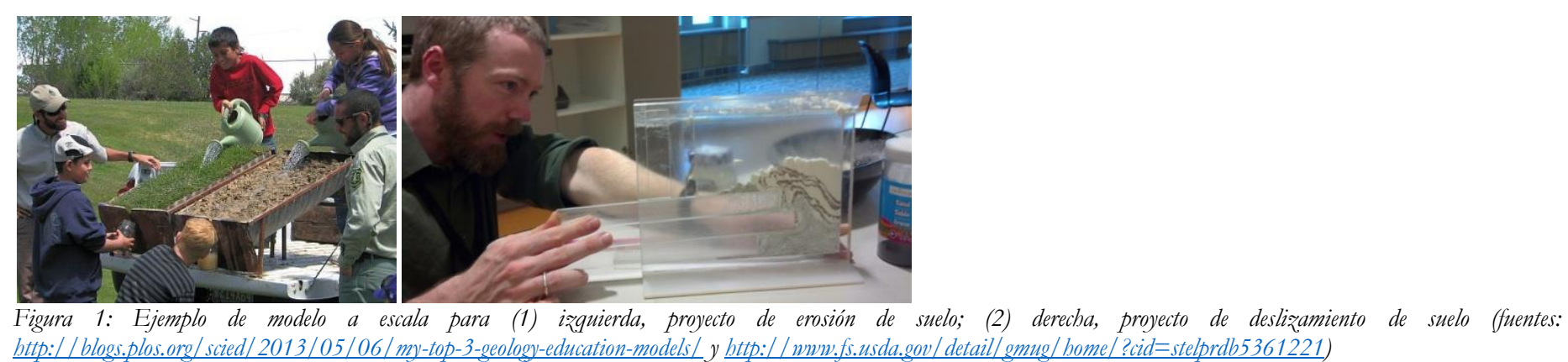

La segunda parte de esta actividad es la grabación del vídeo por los estudiantes con sus smartphones. En caso de no disponer de ellos, se pone a disposición una cámara de vídeo del departamento.

Finalmente, la edición de los vídeos se hace también por los propios estudiantes mediante software libre (Kdenlive, OpenShot o Pitivi).

\section{Actividad 3. Elaboración de la herramienta digitaly portal web.}

Finalmente, los vídeos o los productos finales se suben a las distintas plataformas o redes en distintos formatos. Se pretende reunir vídeos de diferentes temas y asignaturas en la plataforma moodle. Al mismo tiempo, los estudiantes pueden publicar los vídeos en las redes sociales de su elección (Twitter, Periscope, Youtube, Instagram, Pinterest, etc).

\subsection{POBLACIÓN Y MUESTRA}

La muestra ha estado compuesta por un total de 18 estudiantes de la asignatura Hidrología de la EPS Belmez (Universidad de Córdoba), de los cuales 6 eran alumnas y 12 alumnos.

\section{RESULTADOS OBTENIDOS}

Los resultados obtenidos se exponen a continuación, siguiendo la subdivisión en 3 actividades descrita en el apartado sobre materiales y métodos. El grupo de 18 estudiantes se ha subdividido en 3 grupos de 6 personas para estudiar 3 temas diferentes y elaborar 3 vídeos diferentes, cada uno con una duración de aproximadamente 5 minutos. Han sido:

\subsection{EL MOVIMIENTO DEL FRENTE DE HUMEDAD EN DIFERENTES TIPOS DE SUELO}

El primer grupo seleccionó como tema el movimiento del frente de humedad en un medio poroso con diferentes texturas. Elaboraron un dispositivo (Figura 2) de 2 paredes transparentes de vidrio separadas $2 \mathrm{~cm}$. Entre las paredes, se introdujo suelo. Gracias a la transparencia de las paredes, al incorporar agua al suelo, se permite visualizar y grabar perfectamente la infiltración del agua en el suelo en un corte transversal. 


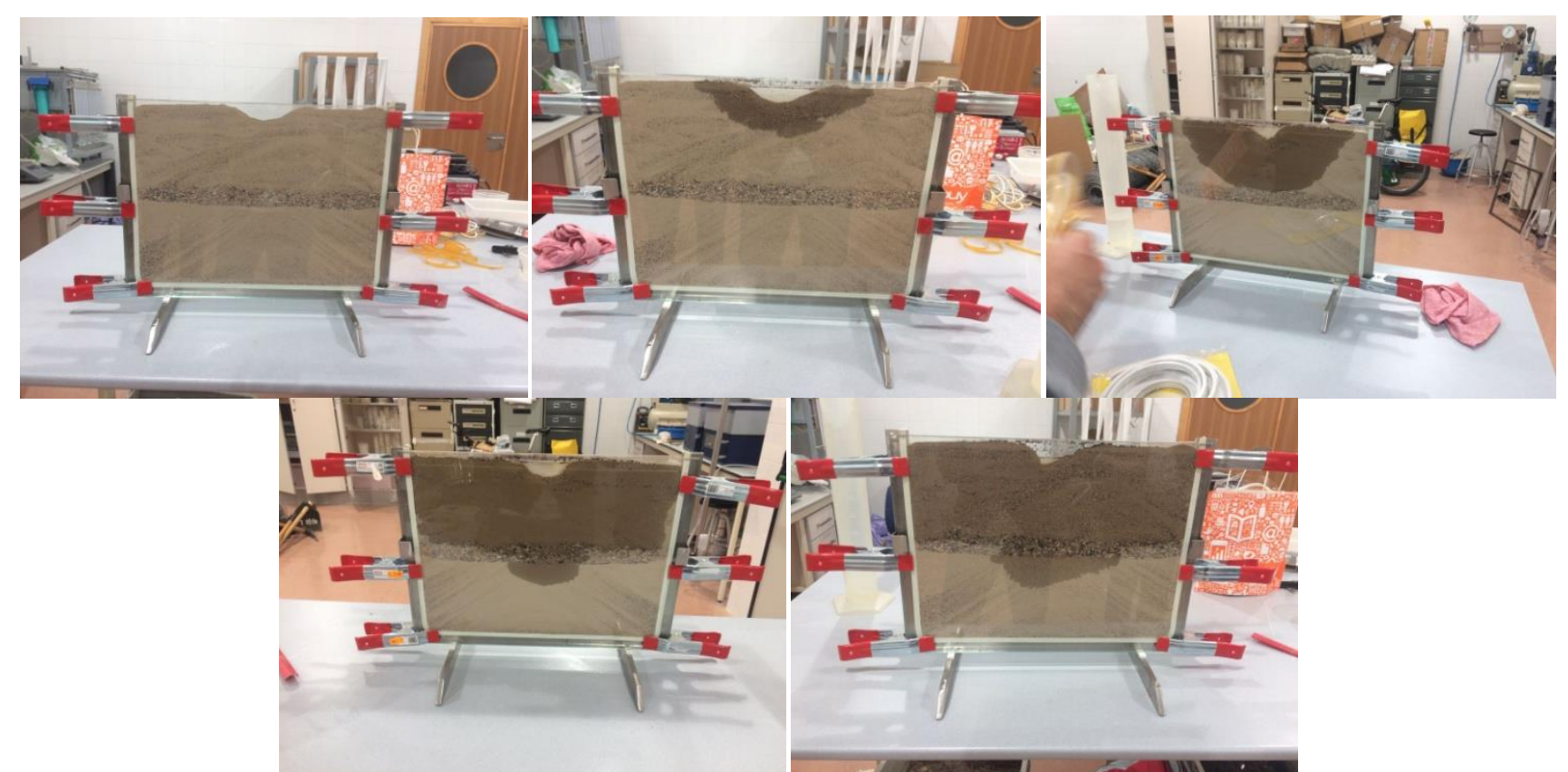

Figura 2: Secuencia tomada del vídeo de movimiento de agua en suelo con diferentes texturas (desde arriba basta abajo) donde se aprecia la infiltración del agua a través de dos capas de arena separadas por una capa de grava.

\subsection{LA RETENCIÓN DE AGUA EN TECHOS VERDES}

El segundo grupo, tras realizar una visita a un techo verde experimental instalado por el Departamento de Agronomía en la Universidad de Córdoba, decidió realizar un modelo a escala de un techo verde para demostrar los beneficios del mismo como son el aumento de la retención de agua por el mismo, evitando posibles inundaciones en las calles; la amortiguación de las oscilaciones de temperatura; y la reducción de la contaminación del agua superficial (Figura 3). El modelo está constituido por 3 compartimentos, en los cuales se pueden instalar 2 techos verdes con diferentes sustratos. En el compartimento central se instaló un techo tradicional como testigo.

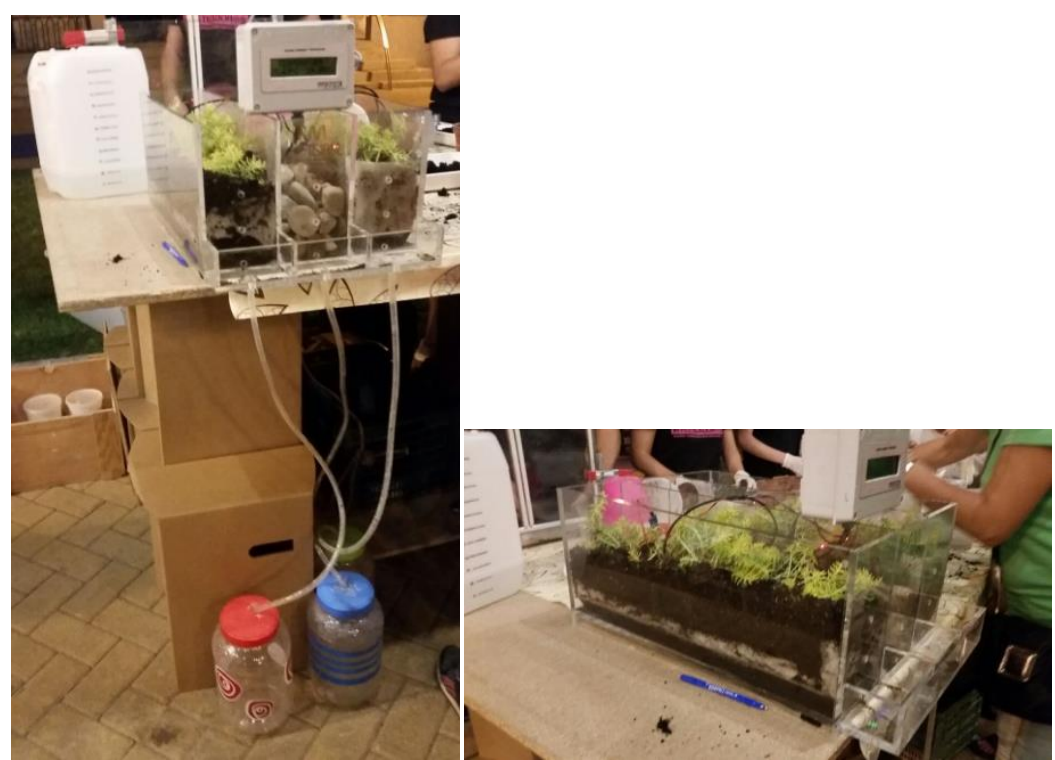

Figura 3: Modelo a escala de techo verde. A la izquierda se aprecia el sistema de recogida del agua de percolación. A la derecha se aprecian las diferentes capas del techo verde.

\subsection{INFILTRACIÓN DE AGUA EN EL SUELO}

El último grupo prefirió grabar y editar un vídeo de una de las prácticas que se realizan en esta asignatura: la medición de la infiltración de agua en el suelo (Figura 4). 


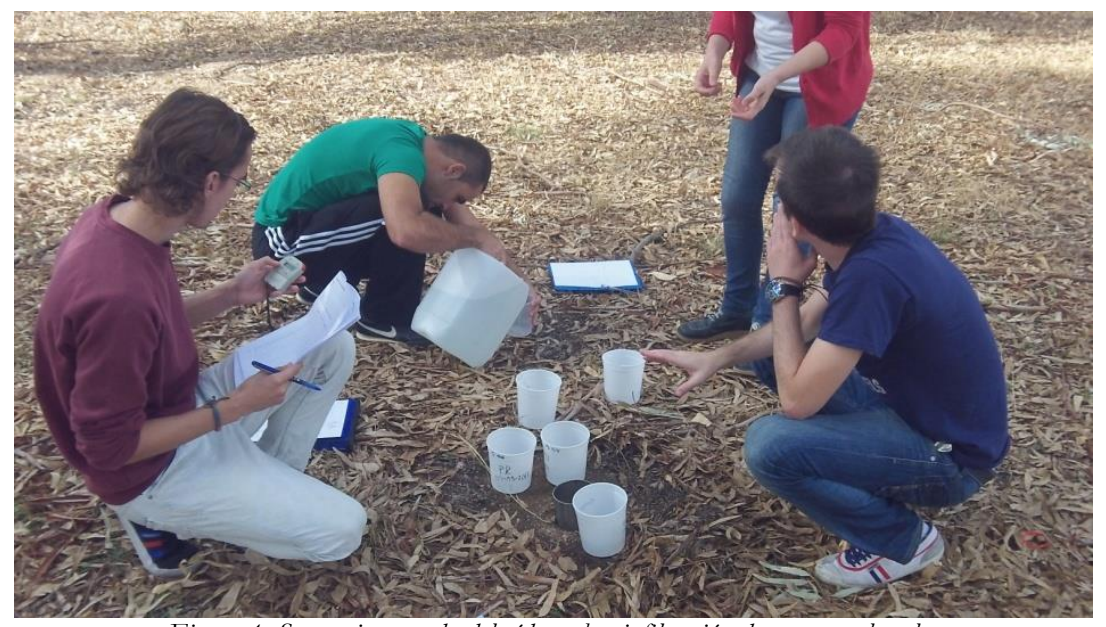

Figura 4: Secuencia tomada del video sobre infiltración de agua en el suelo.

\section{UTILIDAD/ANÁLISIS}

La última fase del estudio ha sido la implicación activa de todos los estudiantes en la enseñanza de la asignatura y la exposición de los tres proyectos para toda la clase. El 100\% de los estudiantes ha evaluado la actividad como muy útil para su aprendizaje (en una escala de 0-10, siendo 10 la nota máxima, la nota media que los estudiantes han dado a esta actividad ha sido de 10). Estos resultados están de acuerdo con los estudios por Felder y col. (1998) y Michaelsen et al. (2004), que demostraron que el aprendizaje colaborativo era superior al tradicional, y que el grupo que había seguido el método colaborativo obtenía mejores notas y un mayor porcentaje de graduados con respecto al grupo de control.

Posteriormente, se han llevado estos vídeos y los modelos a escala que se han producido en el curso de la asignatura (descritos en los puntos 4.1 y 4.2) fuera de las aulas y se han utilizado en las actividades de divulgación "Paseo por la Ciencia", "Feria de los Ingenios" y "Noche de los Investigadores" de la Universidad de Córdoba en el curso académico 2016-2017. Durante estas actividades los modelos también han sido usados por estudiantes de entre 6 y 17 años, con gran éxito (con una estimación de 1.500 impactos).

\section{CONCLUSIONES/DISCUSIÓN}

Resumiendo, en este trabajo se ha puesto en práctica un método de auto-aprendizaje y enseñanza incluyendo la elaboración de modelos a escala de sistemas reales y de vídeos explicativos por parte de los estudiantes. Según nuestras observaciones y seguimiento de la actividad, se puede concluir que esto ha servido para:

- Impulsar la modernización educativa a través de las nuevas tecnologías de la información y de la comunicación que perfeccionen el proceso de aprendizaje-enseñanza, así como para facilitar la comprensión de la materia por parte del alumnado mejorando los conocimientos y por ende los resultados académicos.

- Enlazar la praxis con los conocimientos teóricos mediante una metodología competencial a través de actividades de carácter práctico y el ulterior análisis de datos.

- Desarrollar en el alumnado la capacidad de trabajo en grupo, de forma que asuman roles que faciliten la cohesión social, la responsabilidad laboral o la autonomía e iniciativa, competencias todas necesarias para la realización exitosa de actividades académicamente dirigidas.

\section{AgRADECIMIENTOS}

Los autores agradecen la financiación por parte de la Universidad de Córdoba a través del programa "Proyectos de Innovación Docente concedidos en la Modalidad 1 del Plan de Innovación y Buenas Prácticas Docentes. Curso 2016/2017.”

\section{BIBLIOGRAFÍA}

BRUFFEE, K.A. (1998). Collaborative learning: Higher education, interdependence, and the authority of knowledge. Baltimore, 1998.

FELDER, R.M., FELDER, G.N. \& DIETZ, E.J. “A longitudinal study of engineering student performance and retention. V. Comparisons with traditionally-taught students.", Journal of Engineering Education 1998, 87(4), 469-480.

MICHAELSEN, L.K., KNIGHT, A.B. \& FINK, L.D. (eds.). Team-based learning: A transformative use of small groups in college teaching. Sterling, VA: Stylus, 2004. 